\title{
Lifetime Anxiety Disorders in Women With Bulimia Nervosa
}

\author{
Cynthia M. Bulik, Patrick F. Sullivan, Frances A. Carter, and Peter R. Joyce
}

We examined the prevalence and ages at onset of additional childhood and adult psychiatric disorders in women with bulimia nervosa and evaluated the differential impact of a mood or anxiety disorder on the presentation of bulimia nervosa. One hundred fourteen women participating in a clinical trial of cognitivebehavioral therapy for bulimia nervosa were assessed at pretreatment with structured diagnostic methodology. Although mood disorders were the most frequently occurring additional psychiatric disorder $(75 \%)$, $64 \%$ experienced an additional anxiety disorder. Age at onset of the anxiety disorders was markedly earlier than age at onset of bulimia nervosa or other comorbid conditions. Stratification of the sample on the pres-

A SUBSTANTIAL NUMBER of investigations have examined patterns of concurrent and lifetime comorbidity in women with bulimia nervosa. ${ }^{1-9}$ Most of these studies have focused on the high prevalence of lifetime affective disorders in bulimic women. Fewer have addressed prevalence of anxiety disorders. In studies that have examined ages at onset of additional psychiatric disorders, a consistent finding has emerged-anxiety disorders tend to occur substantially earlier than the onset of bulimia nervosa.

Schwalberg et al. ${ }^{10}$ found that $75 \%$ of women with bulimia nervosa had an additional lifetime diagnosis of an anxiety disorder, and $59 \%$ of these women reported that the age at onset of the anxiety disorder preceded the onset of bulimia. Braun et al. ${ }^{6}$ compared the prevalence of anxiety disorder across subtypes of women with eating disorders and found that more women with concurrent anorexia and bulimia nervosa tended to have an anxiety disorder as their first psychiatric diagnosis, and $37 \%$ of the

From the Department of Psychology, University of Canterbury, Christchurch; and the University Department of Psychological Medicine, Christchurch School of Medicine, Christchurch, New Zealand.

P.F.S. was supported by a research training fellowship from the Health Research Council of New Zealand. This project was supported by grants from the Health Research Council and the Lottery Grants Board.

Address reprint requests to Cynthia M. Bulik, Ph.D., Department of Psychology, University of Canterbury, Private Bag 4800, Christchurch, New Zealand.

Copyright $\odot 1996$ by W.B. Saunders Company

0010-440X/96/3705-0008\$03.00/0 ence of a mood or anxiety disorder revealed no differences in the core bulimic symptoms across groups. The presence of a mood disorder was associated with greater body dissatisfaction, lower Global Assessment of Functioning Scales (GAFS) score, more externalizing disorders of childhood, and, as expected, higher Hamilton Depression Rating Scale (HDRS) scores. The presence of an anxiety disorder was related to a history of anorexia nervosa and earlier age at onset of drug or alcohol dependence. Early-onset anxiety disorders are prevalent and may represent one potential pathway to bulimia nervosa.

Copyright 11996 by W.B. Saunders Company

entire sample had a lifetime anxiety disorder. Brewerton et al. ${ }^{11}$ also found a $36 \%$ prevalence of anxiety disorder in bulimic women, with $71 \%$ showing the onset of anxiety disorders before the onset of bulimia. In a related study, Deep et al. ${ }^{12}$ found that $75 \%$ of 25 long-term weightrecovered anorectics had a lifetime diagnosis of one or more anxiety disorders and that $58 \%$ had the onset of one or more childhood anxiety disorders before age 18. Seventy-nine percent of these women had the onset of the anxiety disorder before the onset of anorexia nervosa. There was no difference in the prevalence of anxiety disorders between restrictor and bulimic anorectics. Taken together, these four studies suggest that early-onset anxiety disorders may be of etiological significance, or may in some way predispose individuals to the development of bulimia nervosa.

The present study was designed to replicate and extend these findings in a sizeable sample of women with bulimia nervosa. All subjects were studied with structured diagnostic methodology. We hypothesized that the majority would report lifetime affective and anxiety disorders and that early-onset anxiety disorders-but not early-onset affective disorders-would be prevalent in women with bulimia nervosa.

\section{METHOD}

The subjects for this study were 114 women aged 17 to 45 years who participated in an outpatient trial of three psychological treatments for bulimia nervosa. ${ }^{13-15}$ All of these women met DSM-III-R ${ }^{16}$ criteria for bulimia nervosa with clearly "objective" binges. ${ }^{17}$ Exclusion criteria were current anorexia nervosa, a body mass index $\leq 17$ or $\geq 30$ $\mathrm{kg} / \mathrm{m}^{2}$, and the current use of psychoactive medication (e.g., 
antidepressants). Three of the subjects presented here were not entered into the treatment study because it was judged clinically that bulimia nervosa was not the principal current diagnosis (one because of current severe major depression and two because of current severe alcohol dependence). The clinical trial was publicized widely in the media and in publications for local eating disorders support groups. Patients were either self-referred or referred from general practitioners and mental health workers. The study had received prior ethical approval and all subjects provided written informed consent.

All subjects underwent a 2- to 4-hour assessment conducted by one of the authors. This assessment consisted of the Structured Clinical Interview for DSM-III-R (SCID) $)^{18}$ which we modified to obtain more information about lifetime history of eating disorders and related behaviors. Age at onset of each of the disorders was established in the "chronology" section of each diagnostic module, which determines the age at onset of each individual disorder. In addition, we assessed the presence of four childhood psychiatric disorders (overanxious disorder, separation anxiety disorder, attention deficit-hyperactivity disorder, and oppositional-defiant disorder). ${ }^{16}$ The presence of these disorders before age 18 was assessed with supplementary modules of the Schedule for Affective Disorders and Schizophrenia-Lifetime Version ${ }^{19}$ written by Kathleen R. Merikangas, Ph.D., and colleagues at Yale University. Only behaviors or symptoms present frequently or constantly, and which clustered in the same time period, were considered to satisfy diagnostic criteria. The interviewing clinician also completed the Structured Clinical Interview for DSM-IIIR-Personality Disorders (SCID-II) ${ }^{20}$ to assess the presence of the 11 DSM-III-R personality disorders. ${ }^{16}$ Conduct disorder was assessed as part of the SCID-II. Finally, the interviewing clinician completed the 17-item Hamilton Depression Rating Scale (HDRS), ${ }^{21}$ Global Assessment of Functioning Scale (GAFS), ${ }^{16}$ and a structured interview that assessed bulimic symptomatology in the prior fortnight including the number of objective binges, the total episodes of purging via vomiting and via laxatives, and the degree of food restriction and body dissatisfaction. All statistical analyses were performed with the JMP statistics package..$^{22}$

\section{RESULTS}

The majority of the present sample of bulimic women met diagnostic criteria for at least one additional lifetime psychiatric disorder besides bulimia nervosa ( $89 \%$ or 102 of 114 ). Mood disorders $(75 \%)$ were the most prevalent comorbid condition (Table 1). Of note, the bipolar II pattern of full depressive and hypomanic episodes was relatively common. Lifetime anxiety disorders (particularly social phobia, simple phobia, and childhood overanxious disorder) were an additional diagnosis in $64 \%$ of these bulimic women. Forty-eight percent of our sample also met criteria for alcohol or drug dependence at some point in their lives. Of
Table 1. Lifetime Psychiatric Disorders in a Cohort of 114 Women with Bulimia Nervosa

\begin{tabular}{|c|c|c|c|}
\hline \multirow[b]{2}{*}{ Disorder } & \multicolumn{2}{|c|}{$\begin{array}{l}\text { Lifetime } \\
\text { Prevalence }\end{array}$} & \multirow{2}{*}{$\begin{array}{l}\text { Age at Onset } \\
\text { (mean } \pm \text { SD) }\end{array}$} \\
\hline & $\%$ & No. & \\
\hline Bulimia nervosa & 100 & 114 & $20 \pm 5$ \\
\hline Anorexia nervosa* & 25 & 28 & $19 \pm 4$ \\
\hline Mood disordert & 75 & 85 & $18 \pm 6$ \\
\hline Major depression & 54 & 62 & $19 \pm 6$ \\
\hline Bipolar II mood disorder & 18 & 20 & $15 \pm 4$ \\
\hline Anxiety disorder $\ddagger$ & 64 & 73 & $8 \pm 7$ \\
\hline Social phobia & 30 & 34 & $11 \pm 5$ \\
\hline Simple phobia & 30 & 34 & $9 \pm 6$ \\
\hline Overanxious disorder & 27 & 31 & $7 \pm 4$ \\
\hline Separation anxiety disorder & 10 & 11 & $7 \pm 4$ \\
\hline Panic disorder & 10 & 11 & $21 \pm 8$ \\
\hline Drug or alcohol dependence & 48 & 55 & $19 \pm 5$ \\
\hline Alcohol dependence & 47 & 54 & $20 \pm 5$ \\
\hline Any drug dependence $\$$ & 22 & 25 & $19 \pm 4$ \\
\hline Childhood externalizing disorder & 26 & 30 & NA \\
\hline Conduct disorder & 23 & 25 & NA \\
\hline Oppositional defiant disorder & 10 & 11 & $9 \pm 3$ \\
\hline $\begin{array}{l}\text { Attention deficit-hyperactivity dis- } \\
\text { order }\end{array}$ & 7 & 8 & $5 \pm 2$ \\
\hline
\end{tabular}

Abbreviation: NA, not available.

*An additional $9 \%$ had subthreshold anorexia nervosa.

How rates of dysthymia $(4 \%, n=4)$ were found. The dysthymia section in the SCID is skipped for subjects with major depression for more than half of the prior 2 years, for those who have ever experienced a manic or hypomanic episode, or those with a chronic psychotic disorder.

‡Low endorsement rates were found for obsessive-compulsive disorder $(4 \%, n=4)$, agoraphobia without panic disorder $(2 \%, n=2)$, and generalized anxiety disorder $(2 \%, n=2)$. As the section on generalized anxiety disorder (GAD) in the SCID is skipped for subjects with a current mood disorder, the rate of GAD may be spuriously low.

$\S$ There was considerable overlap across drug categories. The most prevalent were cannabis dependence $(16 \%, n=18)$ and stimulant dependence $(10 \%, \mathrm{n}=11)$.

interest, the prevalence of lifetime alcohol or drug abuse was quite low suggesting an "all or nothing" approach to substance use in these women. Externalizing disorders of childhood (especially conduct disorder) had been present in $26 \%$ of our sample. Finally, $25 \%$ of these 114 bulimic women had met criteria for anorexia nervosa.

The age-at-onset data in Table 1 clearly suggest that anxiety disorders had the earliest mean age at onset ( 8 years). Panic disorder is a conspicuous exception since its mean age at onset tended to be considerably later (21 years). The mean age at onset of bulimia nervosa ( 20 years), anorexia nervosa (19 years), mood disor- 
der (18 years), and psychoactive substance dependence ( 19 years) tended to be similar.

We then examined the patterns of onset of mood and anxiety disorders relative to bulimia nervosa. The age at onset of two disorders was considered to be contemporaneous if the recorded age at onset of the anxiety or mood disorder was 1 year earlier or later than the age at onset of bulimia nervosa. This allowed for measurement and recall error. A disorder was considered to predate the onset of bulimia if the recorded age at onset was more than 1 year earlier than the onset of bulimia nervosa, and to postdate the onset of bulimia if the recorded age at onset of the disorder was more than 1 year later than the onset of bulimia nervosa. These analyses showed that, for the 85 women with a mood disorder, the mood disorder occurred before bulimia nervosa in $45 \%$, after bulimia nervosa in $16 \%$, and at approximately the same time in $39 \%$. In contrast, in the 73 women with an anxiety disorder, the anxiety disorder occurred before bulimia nervosa in $92 \%$, after bulimia nervosa in $7 \%$, and at approximately the same time in $1 \%$.

The finding of a far earlier onset of an anxiety disorder might be an artifact as two of the anxiety disorders included had, by definition, onset in childhood. When overanxious disorder and separation anxiety disorder were excluded, $53 \%$ of the sample (60 of 114) met criteria for panic disorder, social phobia, simple phobia, obsessive-compulsive disorder, or agoraphobia without panic disorder (AWOPD). Excluding the childhood anxiety disorders, the mean age at onset for anxiety disorder was 9 years (SD, 7 years). Thus, by including the two childhoodonset anxiety disorders in Table 1, the proportion of the sample with an anxiety disorder increased somewhat, but the mean age at onset remained similar.

Mood and anxiety disorders were clearly the most prevalent lifetime conditions comorbid with bulimia nervosa (Table 1). To determine the relative impact of mood and anxiety disorders, we stratified our sample into four groups defined by the presence or absence of a mood or anxiety disorder. Of these 114 bulimic women, $53 \%$ had experienced both a mood and an anxiety disorder, $21 \%$ a mood disorder only, $11 \%$ an anxiety disorder only, and $15 \%$ neither an additional mood nor an anxiety disorder. The odds ratio for the relationship between a mood disorder and an anxiety disorder was significantly increased (odds ratio $=3.60,95 \%$ confidence interval (CI), 1.50 to 8.66 ), suggesting that the presence of one condition was associated with a 3.6 -fold increase in the likelihood of the other. Given its earlier onset (Table 1 ), an anxiety disorder was probably the initial step toward mood-anxiety comorbidity for most of these bulimic women.

We next compared the characteristics of bulimic women stratified into these four groups (Table 2 ). Where the model probability value was statistically significant, differences across groups were further analyzed by fitting a factorial model (analysis of variance or nominal logistic regression where appropriate) with the main effects of a mood disorder, an anxiety disorder, and their interaction.

There were significant differences across groups in the degree of food restriction (greater with a mood or an anxiety disorder), the degree of body dissatisfaction (greater with a mood disorder), total personality disorders and symptoms (greater with a mood or an anxiety disorder), GAFS score (lower with a mood disorder), HDRS total (lower with a mood disorder), age at onset of a mood disorder (earlier with an anxiety disorder), age at onset of any drug/ alcohol dependence (earlier with an anxiety disorder), past history of anorexia nervosa (greater with an anxiety disorder), and presence of any childhood externalizing disorder (greater with a mood disorder) (Table 2). Age, frequency of binging and purging, body mass indices, age at onset of bulimia, age at onset of an anxiety disorder, and history of drug or alcohol dependence were statistically similar across groups. Moreover, in no instance was the interaction term significant, indicating that the influences of these two variables were additive and not synergistic.

Thus, stratification of bulimic women into these four groups tended to identify individuals with differential food restriction, body dissatisfaction, personality pathology, current functioning, age at onset of mood and substance dependence, prior anorexia nervosa, and childhood “externalizing” conditions. 
Table 2. Characteristics of 114 Women with Bulimia Nervosa Stratified Into Groups Defined by the Presence and Absence of Lifetime Mood and Anxiety Disorders

\begin{tabular}{|c|c|c|c|c|c|c|c|c|c|}
\hline \multirow[b]{2}{*}{ Characteristic } & \multicolumn{2}{|c|}{ Mood + Anxiety +} & \multicolumn{2}{|c|}{ Mood+ Anxiety- } & \multicolumn{2}{|c|}{ Mood-Anxiety+ } & \multicolumn{2}{|c|}{ Mood - Anxiety- } & \multirow[b]{2}{*}{$p$} \\
\hline & $\%$ & No. & $\%$ & No. & $\%$ & No. & $\%$ & No. & \\
\hline Group size & & 61 & & 24 & & 12 & & 17 & NA \\
\hline Age $(y r)$ & \multicolumn{2}{|c|}{$26 \pm 6$} & \multicolumn{2}{|c|}{$27 \pm 6$} & \multicolumn{2}{|c|}{$27 \pm 7$} & \multicolumn{2}{|c|}{$27 \pm 7$} & .89 \\
\hline Objective binges/fortnight* & \multicolumn{2}{|c|}{$11 \pm 12$} & \multicolumn{2}{|c|}{$12 \pm 12$} & \multicolumn{2}{|c|}{$11 \pm 11$} & \multicolumn{2}{|c|}{$9 \pm 9$} & .93 \\
\hline Vomiting episodes/fortnight* & \multicolumn{2}{|c|}{$11 \pm 13$} & \multicolumn{2}{|c|}{$15 \pm 13$} & \multicolumn{2}{|c|}{$12 \pm 11$} & \multicolumn{2}{|c|}{$11 \pm 9$} & .56 \\
\hline Laxative use episodes/fortnight* & \multicolumn{2}{|c|}{$2 \pm 5$} & \multicolumn{2}{|c|}{$3 \pm 7$} & \multicolumn{2}{|c|}{$1 \pm 4$} & \multicolumn{2}{|c|}{$1 \pm 1$} & .50 \\
\hline High food restriction $\dagger$ & 62 & 38 & 42 & 10 & 42 & 5 & 24 & 4 & .02 \\
\hline High body dissatisfactiont & 44 & 27 & 54 & 13 & 8 & 1 & 12 & 2 & .004 \\
\hline \multicolumn{10}{|l|}{ Body mass index (BMl $\left.\left[\mathrm{kg} / \mathrm{m}^{2}\right]\right)$} \\
\hline Current BMI & \multicolumn{2}{|c|}{$23 \pm 3$} & \multicolumn{2}{|c|}{$23 \pm 3$} & \multicolumn{2}{|c|}{$23 \pm 2$} & \multicolumn{2}{|c|}{$22 \pm 2$} & .94 \\
\hline Desired BMI & \multicolumn{2}{|c|}{$20 \pm 2$} & \multicolumn{2}{|c|}{$20 \pm 2$} & \multicolumn{2}{|c|}{$20 \pm 1$} & \multicolumn{2}{|c|}{$20 \pm 2$} & .55 \\
\hline Minimum mature BMI & & & & & & & & & .55 \\
\hline Maximum mature BMI & & & & & & & & & .45 \\
\hline Total personality disorders* & & & & & & & & & $<.00005$ \\
\hline Personality disorder symptoms* & & & & & & & & & $<.00005$ \\
\hline GAFS score & & & & & & & & & $<.00005$ \\
\hline HDRS total (17-item) & & & & & & & & & .0001 \\
\hline Age at onset $¥$ & & & & & & & & & \\
\hline Bulimia nervosa & & & & & & & & & .99 \\
\hline Mood disorder & & & & & & & & & .0003 \\
\hline Anxiety disorder & & & & & & & & & .75 \\
\hline Drug/alcohol dependence & & & & & & & & & .04 \\
\hline Drug/alcohol dependence & 57 & 35 & 42 & 10 & 42 & 5 & 29 & 5 & .17 \\
\hline History of anorexia nervosa & 33 & 20 & 13 & 3 & 33 & 4 & 6 & 1 & .05 \\
\hline Any childhood externalizing disorder & 34 & 21 & 29 & 7 & 17 & 2 & 0 & 0 & .03 \\
\hline
\end{tabular}

NOTE. The four groups are defined by the presence $(+)$ or absence $(-)$ of lifetime diagnoses of mood disorder and an anxiety disorder. Values shown are mean $( \pm \mathbf{s d}$ ) or percentage (no.) as indicated. Probability values are from $F$ ratios for continuous variables and $x^{2}$ test for the remainder.

*As the distributions of these variables were nonnormal, Wilcoxon nonparametric comparisons were also performed. Results of parametric and nonparametric analyses were similar.

tDetermined by median split.

¥Probability values from product-limit (Kaplan-Meier) survival analyses.

\section{DISCUSSION}

Results of the present study confirm the high prevalence of additional psychiatric disorders in women with bulimia nervosa and provide insight into the pattern of acquisition of psychiatric disorders in this group. The order of prevalence of additional diagnoses was (1) a mood disorder $(75 \%),(2)$ an anxiety disorder $(64 \%)$, (3) psychoactive substance dependence (48\%), (4) a childhood "externalizing" disorder $(26 \%)$, and (5) anorexia nervosa (25\%). Although both mood and anxiety disorders were present in a clear majority of individuals, marked differences emerged regarding the ages at onset of these disorders. In particular, the mean age at onset of an anxiety disorder was markedly earlier than for bulimia nervosa or a mood disorder. Of the women with additional anxiety disorders, $92 \%$ experienced the anxiety disorder before the bulimia nervosa, whereas $45 \%$ experienced a hypomanic or depressive episode before developing bulimia nervosa.

Unlike previous studies, our definition of "an anxiety disorder" included two childhood anxiety disorders (overanxious disorder and separation anxiety disorder), which were assessed as part of our structured diagnostic interview. Because these two disorders were fairly prevalent and, by definition, began early in life, it is possible that their inclusion in our definition of an anxiety disorder could have artifactually lowered the mean age at onset that we observed. When we excluded the childhood anxiety disorders from the analyses, the overall 
prevalence of anxiety disorders in the sample decreased slightly; however, the mean age at onset remained similar.

These findings confirm and extend prior reports in the literature. , $10-12^{2}$ The findings of a high prevalence of additional anxiety disorders, coupled with their substantially earlier age at onset than affective disorders, suggest that the prior focus on mood disorders may have obscured what may be a more fundamental relation between bulimia nervosa and anxiety disorders. The consistency of this finding supports a model of bulimia nervosa that highlights earlyonset anxiety disorders as one potential pathway to eating disorders. Childhood anxiety disorders and bulimia nervosa could share a biological or temperamental predisposition (e.g., behavioral inhibition or harm avoidance) which could predispose subjects to the development of both disorders. Alternatively, childhood anxiety disorders may be independent conditions from bulimia nervosa that may nonetheless increase vulnerability to developing a later eating disorder. Additional support for this hypothesis is provided by Kendler et al. ${ }^{23}$ who determined that bulimia shared genetic etiological factors with panic disorder and phobias.

Several caveats must be considered when evaluating these results. First, in the absence of a control group we are unable to determine whether the observed patterns of acquisition of disorders are unique to bulimia nervosa, or whether childhood anxiety disorders may predispose subjects to a variety of other psychiatric disturbances. Indeed, a high prevalence of comorbid social phobia (28\%) has also been reported in a sample of individuals with onset of major depression before age 18 . Similar to our results, the anxiety disorders preceded the onset of depression in two thirds of the cases. Thus, one possible interpretation is that individuals with early-onset anxiety disorders may be more prone to comorbidity, and that the order of appearance of the various disorders we observed simply reflects the natural history of the disorders. Second, a large number of women in our sample reported lifetime alcohol dependence $(48 \%)$, which is on the high end of the range reported in other clinical samples in the literature. These rates are high as we did not exclude individuals from the trial with current mild/moderate alcohol dependence. The presence of an anxiety disorder was greater in those individuals with lifetime alcohol dependence $(65 \%)$ than in those with no such history $(42 \%)$. Thus, the particular nature of our sample may have contributed to the observed prevalence of anxiety disorders.

Second, we are constrained by the biases inherent in a referred sample. Although two studies have examined age at onset of additional psychiatric disorders in bulimic women using epidemiological samples, ${ }^{2,4}$ neither has addressed age at onset of anxiety disorders relative to bulimia nervosa. Supporting our methodology, the study of comorbidity in clinical samples is of clinical relevance, although limited in the ability to establish etiology.

Third, differential recall of anxiety and mood disorders could have biased our results. Whereas early anxiety symptoms may be clearly recalled because of their vividness (e.g., fears of speaking in class or fears of separation from an attachment figure), the recollection of depressive symptoms may be less precise. Indeed, many children experience long prodromal dysthymic periods before developing clear major depression. ${ }^{24}$ Arguing against this is the fact that our interviews probed for early onset of all disorders, and diagnoses were only made when sufficiently robust information was provided to make a definite diagnosis.

The second aspect of the study provides a unique way in which to judge the impact of coexisting mood and anxiety disorders in women with bulimia nervosa. As has often been reported with other attempts to stratify bulimic women by the presence or absence of a given disorder or symptom (i.e., sexual abuse, borderline personality disorder, alcohol dependence, or family history), ${ }^{14,15,25,26}$ the core bulimic behaviors did not differ across our four groups defined by the presence or absence of mood or anxiety disorders. Age at onset, body mass index, rates of binging and purging, and laxative abuse were not different across groups. Although exceptions to this finding have been noted, where bulimic women with depression exhibited less vomiting, ${ }^{27}$ and bulimic women with personality disorders used more laxatives, ${ }^{14,25}$ the relative stability of rates of binging and vomiting regardless of stratification, sug- 
gests that bulimia nervosa tends to develop a life of its own. Once established, the core bulimic behaviors remain unaffected by the past or present existence of a variety of symptoms or disorders.

Other significant differences did emerge based on this stratification strategy. The presence of a mood disorder was associated with greater body dissatisfaction, lower GAFS score, more externalizing disorders of childhood, and, as expected, higher HDRS scores. This suggests that body dissatisfaction may be one of the symptoms of bulimia nervosa which is directly related to the presence of mood disturbances and that overall functioning may be most impaired in bulimic women with depression. Indeed, significant associations between symptoms measures of low mood and body image distortion and dissatisfaction have been reported in anorectic, ${ }^{28,29}$ bulimic, ${ }^{30}$ and nonclinical populations. ${ }^{31,32}$ The presence of an anxiety disorder was related to a history of anorexia nervosa and earlier age at onset of drug or alcohol dependence. Finally, the presence of either a mood or an anxiety disorder was associated with greater degrees of food restriction, more personality disorders, and more total personality disorder symptoms.

Overall, these findings indicate that although major depression is the most frequent additional lifetime psychiatric disorder in women with bulimia nervosa, anxiety disorders are present in a substantial number. In addition, anxiety disorders tend to occur significantly earlier than either affective disorders or bulimia nervosa and may represent one pathway into bulimia nervosa. This study has also demonstrated the differential impact of additional affective and anxiety disorders and suggests that, although the presence of these disorders does not alter the core symptoms of bulimia nervosa, other aspects of the disorder and global functioning are affected by the presence of these conditions. Future directions will include determining how the presence of anxiety and affective disorders affects outcome of treatment for bulimia nervosa.

\section{ACKNOWLEDGMENT}

The authors thank the staff of the Bulimia Treatment Programme for their assistance.

\section{REFERENCES}

1. Hudson JI, Pope HG, Yurgelun-Todd D, Jonas JM, Frankenburg FR. A controlled study of lifetime prevalence of affective and other psychiatric disorders in bulimic outpatients. Am J Psychiatry 1987;144:1283-1287.

2. Bushnell JA, Wells E, McKenzie JM, Hornblow AR, Oakley-Browne MA, Joyce PR. Bulimia comorbidity in the general population and in the clinic. Psychol Med 1994;24: 605-611.

3. Yates WR, Sieleni B, Reich J. Comorbidity of bulimia nervosa and personality disorder. J Clin Psychiatry 1989;50: 57-59.

4. Kendler K, MacLean C, Neale M, Kessler R, Heath A, Eaves $\mathrm{L}$. The genetic epidemiology of bulimia nervosa. Am J Psychiatry 1992;148:1627-1637.

5. Mitchell J, Specker S, De Zwaan M. Comorbidity and medical complications of bulimia nervosa. J Clin Psychiatry 1991;52:13-20.

6. Braun D, Sunday S, Halmi K. Psychiatric comorbidity in patients with eating disorders. Psychol Med 1994;24:859867.

7. Bulik C. Drug and alcohol abuse by bulimic women and their families. Am J Psychiatry 1987;144:1604-1606.

8. Hudson J, Pope $\mathbf{H}$, Jonas J, Yurgelun-Todd D. Phenomenologic relationship of eating disorders to major affective disorder. Psychiatry Res 1983;9:345-354.

9. Laessle R, Kittl S, Fichter M, Wittchen H, Pirke K. Major affective disorder in anorexia nervosa and bulimia. $\mathrm{Br}$ J Psychiatry 1987;151:785-789.

10. Schwalberg M, Barlow B, Alger S, Howard L. Com- parison of bulimics, obese binge eaters, social phobics, and individuals with panic disorder on comorbidity across DSMIII-R anxiety disorders. J Abnorm Psychol 1992;101:675681.

11. Brewerton $T$, Lydiard $R$, Herzog $D$, Brotman $A$, O'Neil P, Ballenger J. Comorbidity of axis I psychiatric disorders in bulimia nervosa. J Clin Psychiatry 1995;56: 77-80.

12. Deep A, Nagy L, Weltzin T, Rao R, Kaye W. Premorbid onset of psychopathology in long-term recovered anorexia nervosa. Int J Eating Disord 1995;17:291-298.

13. Carter F, Bulik CM. Exposure treatments for bulimia nervosa: procedure, efficacy, and mechanisms. Adv Behav Res Ther 1994;16:77-129.

14. Bulik C, Sullivan P, Carter F, Joyce P. Temperament, character, and personality disorder in bulimia nervosa. $\mathrm{J}$ Nerv Ment Dis 1995;183:593-598.

15. Sullivan $P$, Bulik $C$, Carter F, Joyce $P$. The significance of a history of childhood sexual abuse in bulimia nervosa. Br J Psychiatry 1995;167:679-682.

16. American Psychiatric Association. Diagnostic and Statistical Manual of Mental Disorders. Ed. 3. Rev. Washington, DC: American Psychiatric Association, 1987.

17. Beglin $S$, Fairburn $C$. What is meant by the term "binge"? Am J Psychiatry 1992;149:123-124.

18. Spitzer R, Williams J, Gibbon M, First M. The structured clinical interview for DSM-III-R (SCID). I. History, rationale, and description. Arch Gen Psychiatry 1992;49:624-629. 
19. Endicott J, Spitzer R. Schedule for Affective Disorders and Schizophrenia-Lifetime Version. New York, NY: New York State Psychiatric Institute 1978;837-844.

20. Spitzer R, Williams J, Gibbon M. Structured clinical interview for DSM-III-R: Personality Disorders (SCID-II). New York, NY: New York State Psychiatric Institute, 1987.

21. Hamilton M. A rating scale for measuring depression. J Neurol Neurosurg Psychiatry 1960;23:56-62.

22. SAS. JMP. Ver. 3. Cary, NC: SAS Institute, 1994.

23. Kendler K, Walters E, Neale M, Kessler R, Health A, Eaves L. The structure of the genetic and environmental risk factors for six major psychiatric disorders in women: phobia, generalized anxiety disorder, panic disorder, bulimia, major depression and alcoholism. Arch Gen Psychiatry 1995;52:374-383.

24. Kovacs M, Akiskal H, Gastonis C, Parrone P. Childhood-onset dysthymic disorder. Arch Gen Psychiatry 1994; 51:265-374.

25. Johnson C, Tobin D, Enright A. Prevalence and clinical characteristics of borderline patients in an eating disordered population. J Clin Psychiatry 1989;50:9-15.

26. Mitchell JE, Hatsukami DK, Pyle RL, Eckert ED.
Bulimia with and without a family history of depressive illness. Compr Psychiatry 1986;27:215-219.

27. Hatsukami D, Mitchell JE, Eckert E, Pyle R. Characteristics of patients with bulimia only, bulimia with affective disorder, and bulimia with substance abuse problems. Addict Behav 1986;11:399-406.

28. Garfinkel P, Garner D. Perceptions of the body in anorexia nervosa. In: Pirke K, Ploog D (eds): Psychobiology of Anorexia Nervosa. Berlin, Germany: Springer Verlag, 1984;136-147.

29. Strober $\mathbf{M}$. The relation of personality characteristics to body image disturbances in juvenile anorexia nervosa: a multivariate analysis. Psychosom Med 1981;43:323-330.

30. Lindholm L, Wilson G. Body image assessment in patients with bulimia nervosa and normal controls. Int J Eating Disord 1988;7:527-539.

31. Cooper P, Bowskill R. Dysphoric mood and overeating. Br J Clin Psychol 1986;25:155-156.

32. Thompson J, Thompson C. Body size distortion and self-esteem in asymptomatic, normal weight males and females. Int J Eating Disord 1986;5:1061-1068. 